\title{
Experimental and Numerical Small-Signal Analysis of Two Types of Gain-Clamped Semiconductor Optical Amplifiers
}

\author{
Sam Verspurten, Student Member, IEEE, Geert Morthier, Senior Member, IEEE, and Roel Baets, Senior Member, IEEE
}

\begin{abstract}
The behavior of two types of gain-clamped semiconductor optical amplifiers under small-signal optical modulation is analyzed both numerically and experimentally. The small-signal gain as well as the crosstalk is investigated in two different injecting schemes. Dependence on mean input power and signal frequency is studied. The study reveals a strong dependence of the small-signal gain on the direction of the data signal as compared to the pump signal. The biggest difference between both amplifier types is found in the co-propagation scheme where the RF gain shows a clear resonance in the case the gain clamping is achieved by a longitudinal laser field which results in a decrease of the small-signal gain around the resonance frequency. The behavior of the crosstalk is similar in both cases, however still showing differences due to the origin of the gain clamping, which will be discussed. The experiments confirm the results found in the numerical study in all cases.
\end{abstract}

Index Terms-All-optical devices, gain-clamped semiconductor optical amplifier (GCSOA), linear optical amplifier, SOA, smallsignal analysis.

\section{INTRODUCTION}

$\mathbf{T}$ HE high crosstalk between data signals propagating simultaneously through a semiconductor optical amplifier (SOA) due to the power dependent gain lead to the development of linear optical amplifiers. Up till now two types of integrated linear optical amplifiers exist, each of them based on the gain clamping by an internal laser cavity. The first type, the so-called gain-clamped SOA (GCSOA) [1], uses a longitudinal laser cavity and is schematically depicted in Fig. 1(a). The second type was given the name linear optical amplifier (LOA) and uses a vertical laser cavity to obtain the gain clamping [2]. The structure of this component is shown in Fig. 1(b). Both components proved their potential in a number of high-speed applications both using the linear (e.g., [1], [3]-[5]) and the nonlinear properties (e.g., [6]-[8]).

So far, for the GCSOA a few studies have been done concerning the dynamic behavior under small-signal optical modulation. In [9] a theoretical small-signal study was reported, introducing a resonance phenomenon in the crosstalk under large

Manuscript received June 22, 2005; revised December 8, 2005. This work was supported in part by the Belgian Research Office BELSPO through the IAP5/18 project and by the European Commission through the Network of Excellence e-PHOTON/ONe. The work of S. Verspurten was supported in part by the Institution for the Promotion of Innovation by Science and Technology (IWT), Flanders.

The authors are with Department of Information Technology, Ghent University, 9000 Ghent, Belgium (e-mail: sam.verspurten@intec.ugent.be).

Digital Object Identifier 10.1109/JQE.2005.864154 signal modulation. In [3] on the other hand, the usability of the component for CATV networks was investigated theoretically, and partially experimentally. Studies investigating the laser power modulation under optical modulation were also presented in order to determine the intrinsic parameters of the laser diode [10]. However no thorough counter- or copropagating crosstalk measurements were performed in the small-signal optical modulation regime.

In the case of the LOA, the crosstalk under large signal modulation was briefly studied in [11]. A small-signal analysis was performed in [12] where a resonance behavior was reported for the crosstalk of a copropagating probe signal, which was confirmed by a few experiments in [13]. However, no systematic or in-depth investigation of the complex small-signal dynamics of both GCSOAs and LOAs has been reported so far.

In this paper, we will discuss the results of a thorough study of the crosstalk in these amplifiers, based on experiments and numerical simulations in two different regimes. Also the influence on the small-signal gain of the injected signal will be investigated. According to our knowledge this hasn't been reported yet. We believe those studies can be helpful to further understand the dynamical behavior of the given components.

The paper is organized as follows. In Section II, a model we developed to simulate the LOA will be briefly described, together with an existing model used for the GCSOA simulations. Section III will present simulation results on the small-signal gain felt by an RF signal co- and counter-propagating with a strong continuous-wave (CW) pump signal in both components. Similarities and differences will be discussed and explained. The next section will report on the simulation results of the crosstalk felt by a forward and backward propagating probe signal in both regimes. Finally, we will compare the numerical results to measurements showing good qualitative agreement with the numerically observed effects. It was not possible to make a quantitative comparison due to the unavailability of the parameters of the devices used in the experiments.

\section{Models USED FOR SimULATIONS}

The numerical study of the LOA was performed with a rate equation based model similar to those developed in [11] and [14]. A wavelength independent gain relation was used, combined with the ultrafast nonlinear gain suppression, mainly caused by spectral hole burning and carrier heating [15]. Random noise sources were not included, since they are not relevant in our study. The multisegmental character (segment 


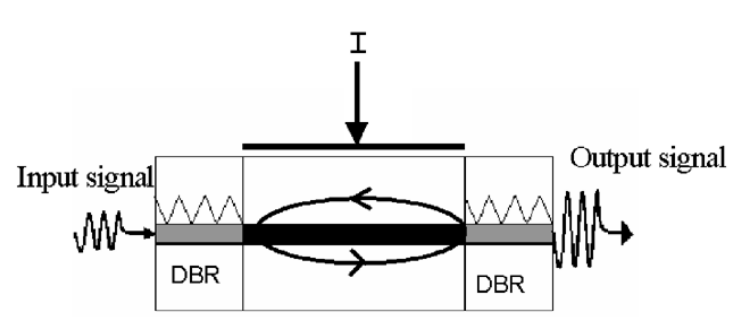

(a)

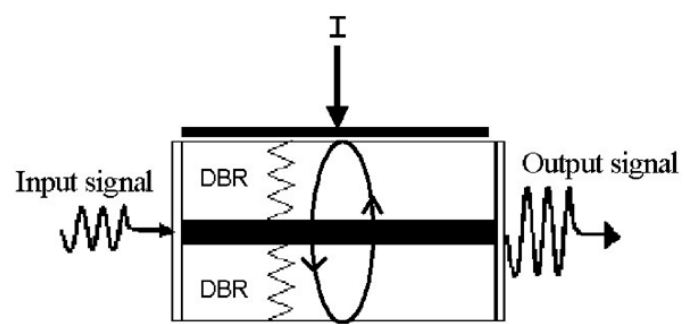

(b)

Fig. 1. Schematic structure of both components studied in this paper. (a) GCSOA. (b) LOA.

TABLE I

Physical Parameters of the LOA USED IN THE SimUlations

\begin{tabular}{lll}
\hline \hline Parameter Notation & Value & Description \\
\hline$\tau_{p h}$ & $10^{-12} \mathrm{~s}$ & Photon lifetime [14] \\
$\mathrm{A}$ & $1.66 \times 10^{8} \mathrm{~s}^{-1}$ & Nonradiative recombination constant \\
$\mathrm{B}$ & $10^{-10} \mathrm{~cm}^{3} \mathrm{~s}^{-1}$ & Bimolecular recombination constant \\
$\mathrm{C}$ & $3 \times 10^{-29} \mathrm{~cm}^{6} \mathrm{~s}^{-1}$ & Auger recombination constant \\
$\mathrm{d}$ & $.2 \mu \mathrm{m}$ & Active layer thickness \\
$\mathrm{W}$ & $1 \mu \mathrm{m}$ & Active layer width \\
$\mathrm{L}$ & $1200 \mu \mathrm{m}$ & LOA length \\
$\Gamma_{s i g}$ & .45 & Confinement factor signal \\
$\Gamma_{L}$ & .65 & Confinement factor laser light \\
$\alpha_{\text {int }}$ & $26.8 \mathrm{~cm}^{-1}$ & Effective loss coefficient \\
$\beta_{s p}$ & $1 \times 10^{-4}$ & Spontaneous emission coupling coefficient \\
$N_{0}$ & $1 \times 10^{18} \mathrm{~cm}^{-3}$ & Transparency carrier density \\
$\mathrm{a}$ & $2.7 \times 10^{-16} \mathrm{~cm}^{2}$ & Differential gain coefficient \\
$\nu_{s i g}$ & $193.55 \mathrm{THz}$ & Signal frequency \\
$\nu_{L}$ & $192 \mathrm{THz}$ & Laser frequency \\
$v_{g}$ & $8.33 \times 10^{7} \mathrm{~ms}^{-1}$ & Group velocity \\
$\varepsilon_{\text {sig }}$ & $3.885 \mathrm{~W}^{-1}$ & Gain suppression coefficient [17] \\
I & $175 \mathrm{~mA}$ & Injected current \\
\hline
\end{tabular}

length approximately $10 \mu \mathrm{m}$ ) enabled a.o. modeling of spatial hole burning effects. Input signals could be injected on both sides of the component. The model is described by the following equations:

$$
\begin{aligned}
\frac{\partial N(z, t)}{\partial t}= & \frac{1}{q} \cdot \frac{I}{w \cdot d \cdot L}-A \cdot N(z, t) \\
& -B \cdot N^{2}(z, t)-C \cdot N^{3}(z, t) \\
& -\frac{\Gamma_{\mathrm{sig}} \cdot g(z, t)}{w \cdot d} \cdot \sum_{i} \frac{1}{h \cdot \nu_{i}} \cdot P_{i}^{ \pm} \\
& -\frac{\Gamma_{L} \cdot g(z, t)}{w \cdot L} \cdot \frac{1}{h \cdot \nu_{L}} \cdot P_{L}(1)
\end{aligned}
$$

$\frac{1}{v_{g}} \cdot \frac{\partial P_{i}^{ \pm}(z, t)}{\partial t} \pm \frac{\partial P_{i}^{ \pm}(z, t)}{\partial z}=\left(\Gamma_{\mathrm{sig}} \cdot g(z, t)-\alpha_{\mathrm{int}}\right) \cdot P_{i}^{ \pm}(z, t)$

$$
\begin{aligned}
\frac{\partial P_{L}(z, t)}{\partial t}= & \left(\Gamma_{L} \cdot g(z, t) \cdot v_{g}-\frac{1}{\tau_{p h}}\right) \\
& \cdot P_{L}(z, t)+\beta \cdot V_{a c t} \cdot B \\
& \cdot N^{2}(z, t) \cdot \frac{v_{g}}{2 \cdot L} \cdot h \cdot \nu_{L}
\end{aligned}
$$

with

$g(z, t)=a \cdot\left(N(z, t)-N_{0}\right)$

$\left.\left.\cdot\left(1-\varepsilon_{\mathrm{sig}} \cdot \Gamma_{\mathrm{sig}} \cdot P_{\mathrm{sig}}(z, t)\right)-\varepsilon_{\mathrm{sig}} \cdot \frac{d}{L} \cdot \Gamma_{L} \cdot P_{L}(z, t)\right)\right)$.

It should be noted that the physical structure of the LOA is reflected in a separate laser field equation for each segment. Table I lists the meaning of different parameters used in the
TABLE II

Physical PARAMETERS OF THE GCSOA USED IN THE SiMUlations

\begin{tabular}{ll}
\hline \hline Description & Value \\
\hline Length active region & $1000 \mu \mathrm{m}$ \\
Length left reflector & $400 \mu \mathrm{m}$ \\
Length right reflector & $400 \mu \mathrm{m}$ \\
Width & $2 \mu \mathrm{m}$ \\
Thickness & $.15 \mu \mathrm{m}$ \\
Injected current & $120 \mathrm{~mA}$ \\
Gain suppression coefficient & $2.59 W^{-1}$ \\
\hline
\end{tabular}

equations, together with the typical values used in the simulations [16].

The GCSOA was studied using the computer model CLADISS [18], a longitudinal multimodal model for the analysis of the static, dynamic and stochastic regime of laser diodes with distributed feedback. A GCSOA could be modeled by surrounding an active section by two passive distributed Bragg reflective (DBR) sections. The laser model also offered the possibility of injecting signals at both facets. The material parameters were chosen as in [19]. The dimensions together with the gain suppression factor are given in Table II. Note that the gain suppression factors of GCSOA and LOA are identical if expressed in $\mathrm{cm}^{3}$ (i.e., $8.8 \times 10^{-18} \mathrm{~cm}^{3}$ ).

\section{SMALL-SignAl AMPLIFICATION UNDER CW INJECTION}

A first study consisted of the determination of the amplification of a RF component superposed on a CW signal. Further on, this will be referred to as the copropagation setup. The dependence on RF frequency and CW input power was investigated. 


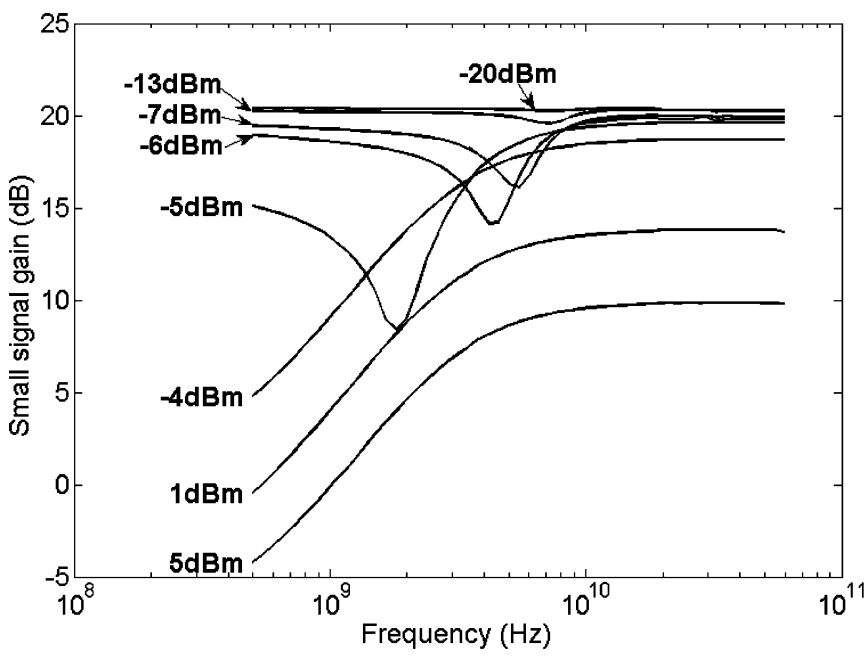

(a)

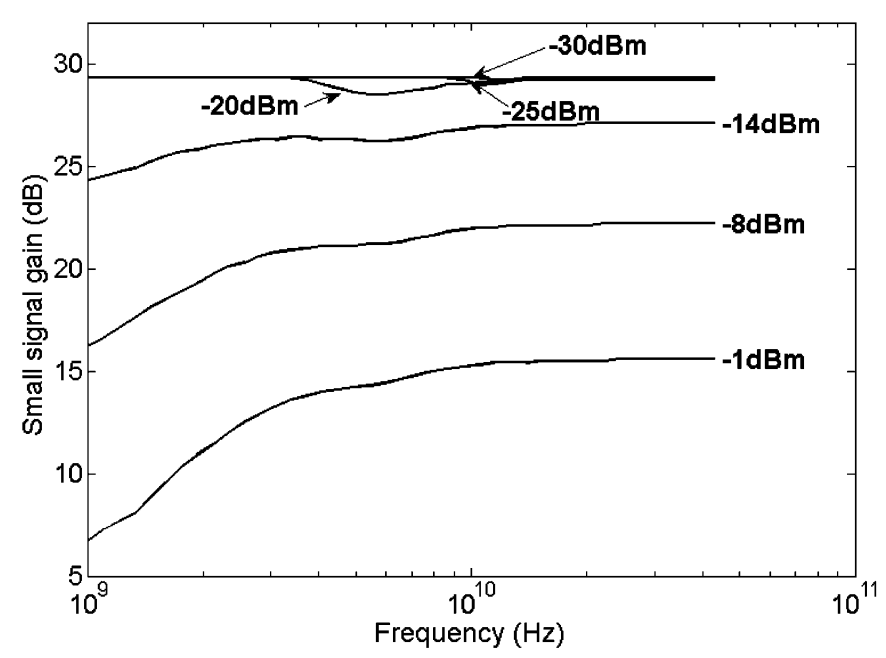

(b)

Fig. 2. Small-signal gain in the copropagation regime for different $\mathrm{CW}$ input powers resulting from the simulation of (a) a GCSOA driven with $120 \mathrm{~mA}$ and (b) a LOA driven with $175 \mathrm{~mA}$.

The small-signal gain is defined as the ratio of the output amplitude and the input amplitude, which was chosen to be $10 \%$ of the CW input power. In Fig. 2(a) the simulated small-signal gain is depicted for a GCSOA driven with a current of 120 $\mathrm{mA}$, for different values of the $\mathrm{CW}$ input power. At low input powers, as compared to the input saturation power (approximately $-4.5 \mathrm{dBm}$ ), a fairly constant amplification as a function of frequency is obtained. The gain remains clamped to the $\mathrm{CW}$ gain under all excitation frequencies. A look into the component showed that for all frequencies, apart from a little spatial hole burning in the front and the back of the amplifier, the gain is equal along the entire length of the GCSOA. At input powers in the vicinity of, but smaller then the input saturation power, a clear resonance phenomenon is observed, resulting in a decrease in small-signal gain. This can be explained by the results of a one sectional small-signal analysis neglecting the gain suppression, as has been done also in [9]. This reveals following relation for the small-signal gain:

$$
\begin{aligned}
& \frac{\Delta S_{\mathrm{sig}}(z=L)}{\Delta S_{\mathrm{sig}}(z=0)}=G_{\mathrm{sig}}^{0}(1+ S_{\mathrm{sig}}(z=0) \cdot \frac{L}{v_{g}} \cdot \Gamma \cdot a \\
&\left.\cdot\left[\frac{j \cdot \Omega \cdot K}{\left(\Omega^{2}-\Omega_{R}^{2}\right)-j \cdot \Omega \cdot \gamma}\right]\right)
\end{aligned}
$$

where $\Omega$ is the frequency of the applied sine, $K$ a constant proportional to $G_{\text {sig }}^{0}$ which is the $\mathrm{CW}$ signal gain per second. $\Omega_{R}$ and $\gamma$ are the resonance frequency and the damping of the incorporated laser under injection of a signal. The expression within square brackets is the carrier density variation averaged over the length of the component divided buy the input signal amplitude. It can be seen that at $\Omega_{R}$, the variation of the carrier density is maximal, while being in antiphase with the input signal variations. This renders the dip in the AM gain. In our simple analysis $\Omega_{R}$ is proportional to the average laser power remaining in the cavity. This explains the shift of the resonance phenomenon to lower frequencies with increasing input power. This was the case for all the currents applied in our study (up to $300 \mathrm{~mA}$ ). This shows that the reduction of $\Omega_{R}$ by the decrease of the laser power was dominant over the possible increase of $\Omega_{R}$ due to the self- and cross-gain saturation effects [10].

The higher carrier density variations when less laser power is present result in an increasing dip depth of the AM gain. This also shows that even if there is not a clear maximum in the laser power oscillation any more due to an increased damping, there still exists a clear maximum in the carrier density variations, thereby still inducing a clear minimum in the small-signal gain. Once we exceed the input saturation power, we immediately get the well-known high-pass characteristic of an SOA [20]. At intermediate input power, slightly lower than the input saturation power, a gradual transition takes place from the resonance regime to the SOA-regime. It should be noted that in any case the low frequency limit is formed by the derivative of the gain versus input power, whereas the high frequency limit equals the $\mathrm{CW}$ gain.

Fig. 2(b) shows corresponding results for a LOA driven with $175 \mathrm{~mA}$. We observe different regimes depending on the input power. However, due to the different nature of the component, significant differences occur as compared to the GCSOA case. To understand the following, it is important to note that a LOA can be seen as a concatenation of "independent" lasers. With increasing input power, subsequently lasers will be quenched, starting at the back of the LOA. At input powers for which the lasing is still present over the entire length of the component (e.g., $-30 \mathrm{dBm}$ ) we get an almost constant amplification in function of frequency. Once lasing stops in a part of the LOA (e.g., $-20 \mathrm{dBm}$ ) the resonating lasers with only a little laser power remaining in the cavity again start to influence the small-signal gain. The strength of the resulting dip however is much smaller as compared to the GCSOA, because it is the result of several oscillating laser sections, each providing only a small part of the total gain. Detailed inspection however again revealed an increasing dip depth and a very small decrease of the resonance frequency with increasing input power. The increasing dip depth mainly originates from the increasing number of oscillating sections which are close to cut-off, together with the increased amplitude of the carrier density os- 


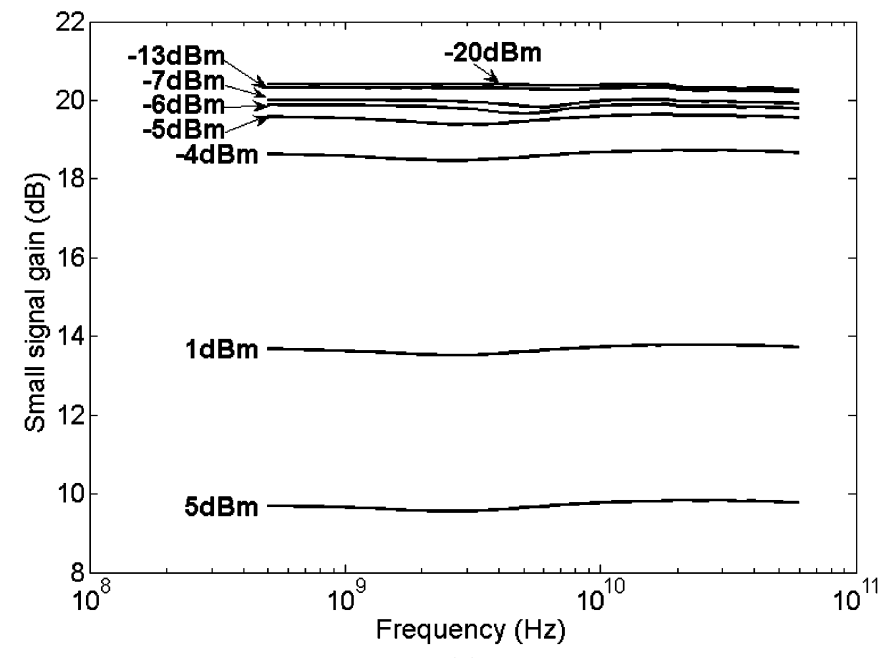

(a)

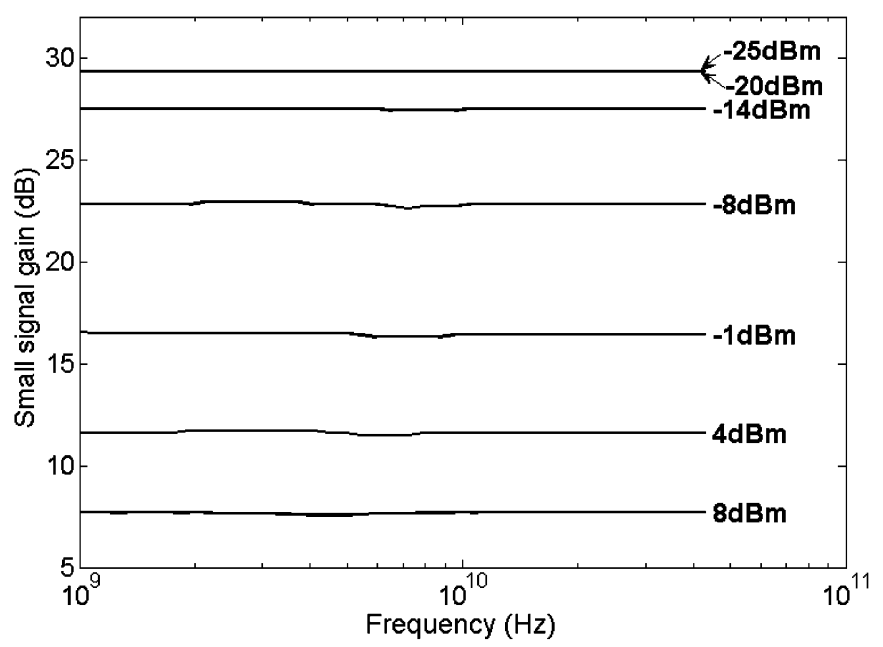

(b)

Fig. 3. Simulated small-signal gain in the counter-propagation regime for different $\mathrm{CW}$ input powers injected in (a) a GCSOA driven with $120 \mathrm{~mA}$ and (b) a $\mathrm{LOA}$ driven with $175 \mathrm{~mA}$. In (b) the curves corresponding with $-20 \mathrm{dBm}$ and $-25 \mathrm{dBm}$ coincide.

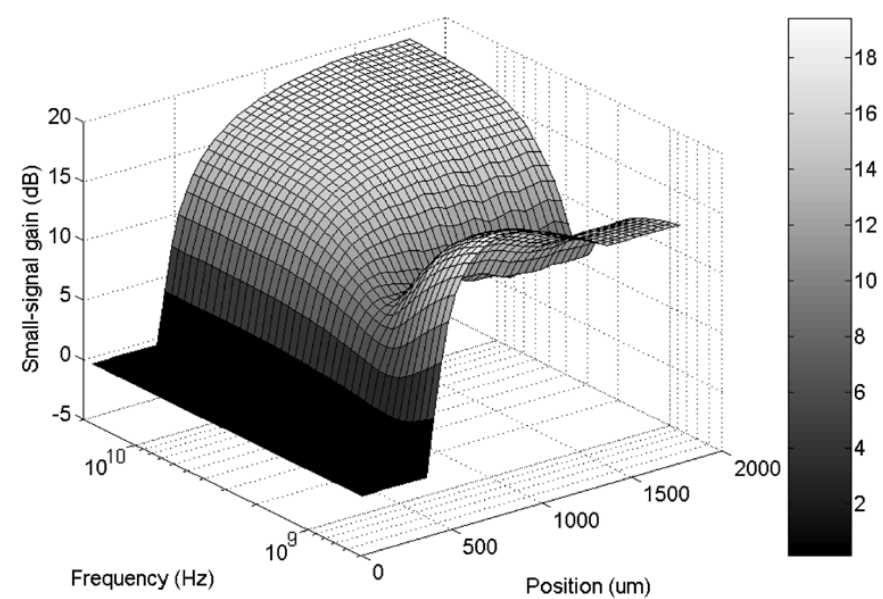

(a)

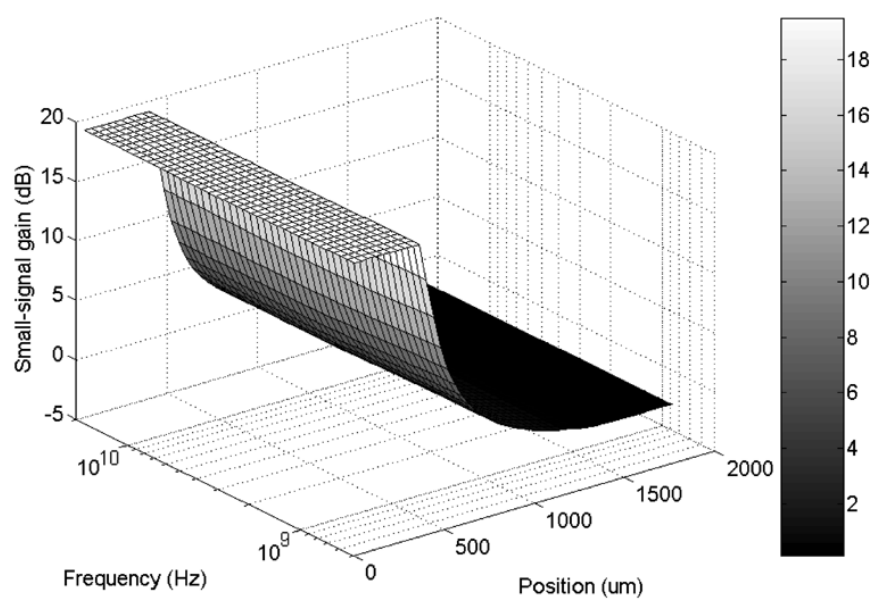

(b)

Fig. 4. Evolution of the amplitude of the injected sine (expressed in dB relative to input amplitude) versus length in the (a) copropagation case and (b) counter-propagation case for a GCSOA with an injected $\mathrm{CW}$ power of $-5 \mathrm{dBm}$. Notice that in the copropagating case the signal is injected from the left hand side and the gain increases from left-hand side to right hand side, while in the counter-propagating case it is the other way around. In both cases however, the amplifier is saturated in the back part.

cillations. Once a significant part of the component stopped lasing, this part acts as an SOA, with an associated high-pass nature. This causes the drop in gain of the low frequencies at higher input powers. There exists a gradual transition to the pure high-pass SOA characteristic. The input power range in which the transition takes places is much larger than in the GCSOA case.

As a conclusion we can state there exists a frequency dependent amplification of a sinusoidal signal copropagating with a CW pump signal in a GCSOA as well as in a LOA. The resonance phenomena of the incorporated clamping mechanisms, leading to a decrease in gain around this resonance frequency, gets more important in the vicinity of the input saturation power, but is less pronounced in a LOA due to the distributed origin of the clamping mechanism.

If the CW and the sinusoidal component are injected from opposite facets of the amplifiers, a different behavior is observed. We studied this counter-propagating regime by injecting a $\mathrm{CW}$ signal at the left facet, combined with a raised sine signal with an amplitude equal to $10 \%$ of the $\mathrm{CW}$ input power at the right facet. This regime will be referred to as the counter-propagation setup. The backward small-signal gain is again defined as the ratio of the output amplitude to the input amplitude of the injected sine. In Fig. 3(a) the simulated gain is depicted in the case of an identical GCSOA as used above. For small CW input powers we observe again a frequency independent small-signal frequency response (SSFR), due to the big reservoir of laser power stored in the cavity. At input powers in the vicinity of the input saturation power (e.g., $-5 \mathrm{dBm}$ ) we observe an almost constant small-signal gain relation, be it with a very little resonance. The high frequency limit remains the $\mathrm{CW}$ gain as was the case for the forward sine. The low frequencies however are inducing a relatively small laser power oscillation, as compared to the copropagation case. This is caused by the smaller amplitude of the sine in the part of the amplifier where gain saturation already exists being most sensitive to input power variations. This can be understood by comparing Fig. 4(a) and (b). In Fig. 4(a), we see a clear resonance combined with low frequency absorption 


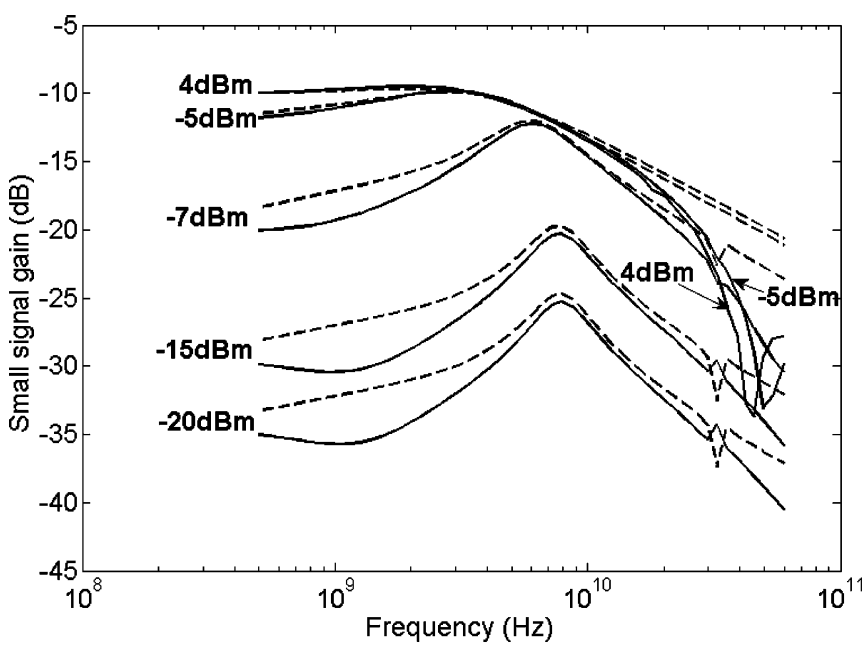

(a)

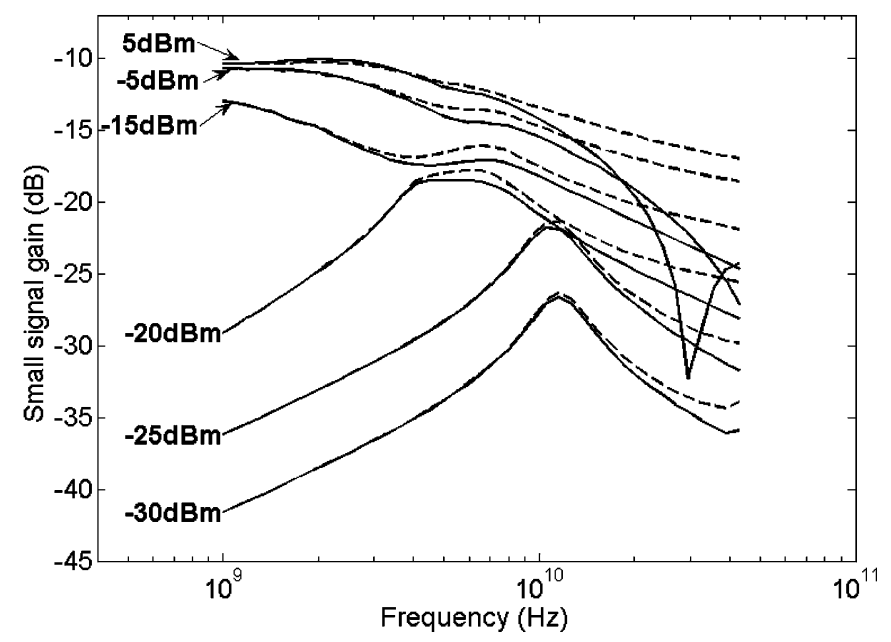

(b)

Fig. 5. Simulated crosstalk induced on a weak probe signal of $-50 \mathrm{dBm}$ in the copropagation regime for different CW input powers in the case of (a) the GCSOA and (b) of the LOA. Full lines and dashed lines correspond to backward and forward propagating probes, respectively.

occurring in the right, gain flattening part of the component. In Fig. 4(b), on the other hand, we can see that in this part the backward propagating signal still has a very small amplitude, which only becomes significant in the less saturated front part of the component. The difference in laser modulation amplitude induced by co- and counter-propagation was logically the biggest for input powers in the transition zone mentioned in the copropagation analysis. For the same reason, also in the SOA regime we get a fairly constant SSFR, equal to the $\mathrm{CW}$ gain.

In the case of the LOA we find similar results. Simulation results for a LOA driven with $175 \mathrm{~mA}$ are depicted in Fig. 3(b). The lack of a clear resonance in any case is even more logical in this case, since the amplitude of the excitation in the vicinity of the oscillating laser sections is much smaller as compared to the forward case.

We can conclude that in both amplifiers an almost frequency independent SSFR is obtained for a counter-propagating RF signal. Even if the gain clamping is quenched by the $\mathrm{CW}$ input power, the traditional high power characteristic of an SOA is equalized.

\section{SMall-Signal Crosstalk Analysis UNDER CW INJECTION}

Although the GCSOAs were developed to reduce interchannel crosstalk, gain fluctuations always will exist under dynamic conditions. Moreover a clear frequency dependence is observed as mentioned in the introduction. A more in-depth study consisting of simulations and experiments will now be given. It is worth noting that in the case of the probe signal we will always talk about the forward and backward propagating probe, whereas for the signal and the pump we keep using the co- and counter-propagation terminology as introduced above.

A first study consisted of the determination of the amount of cross gain modulation in forward and backward direction under the co-propagation regime studied above. This was simulated by injecting a very weak probe signal with a constant optical power of $-50 \mathrm{dBm}$ at each facet of the component in addition to the data signals. The wavelength was chosen $3 \mathrm{~nm}$ smaller then the signal wavelength. The small-signal crosstalk was defined as the ratio of the amplitude of the probe output power variations to the mean probe output power, in accordance to [2].

For the same GCSOA as studied above, Fig. 5(a) depicts the crosstalk felt by a forward and a backward propagating probe under the copropagating setup described in Section III. In the case of significant remaining laser power a resonance is observed, leading to a maximum in the gain modulation. This could also be concluded from a part of (5) since the modulation of the probe is proportional to the carrier density modulation which can be found between the square brackets. This resonance was predicted theoretically for a forward propagating signal under large signal modulation in [9] and for small-signal modulation in [3]. We observe a very low crosstalk for both high and low frequencies. At low frequencies, the laser power oscillation is approximately in antiphase with the input signal modulation, rendering only a small change in the average power in the cavity. At high frequencies on the other hand, the carrier density and the laser power cannot follow the input signal variations any more, due to the finite electron lifetime. At high input powers, we observe the convergence to the low-pass characteristic of a SOA, showing a small overshoot due to the finite loss in the cavity [21]. It can be seen that this crosstalk is always larger than in any gain-clamped regime.

Inspecting the crosstalk on the backward probe it could be remarked that in any case this is smaller as compared to the forward crosstalk. This could be intuitively understood by the fact that the forward propagating probe signal is constantly feeling the source of the gain modulation. At very low frequencies, this is also the case for the backward propagating probe, which results in an identical low frequency limit for both propagation directions of the probe. As the signal frequency increases however, a phase difference exists between the local carrier density modulation (due to the signal and forward and backward propagating laser powers) and the probe modulation (already accumulated during earlier propagation). This results in a difference between the crosstalk in both directions. A similar difference 


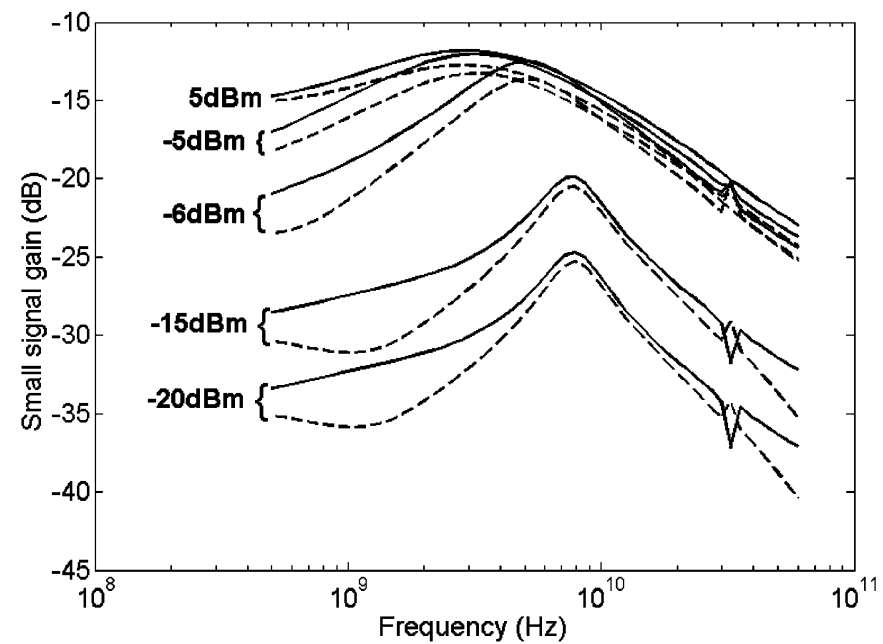

(a)

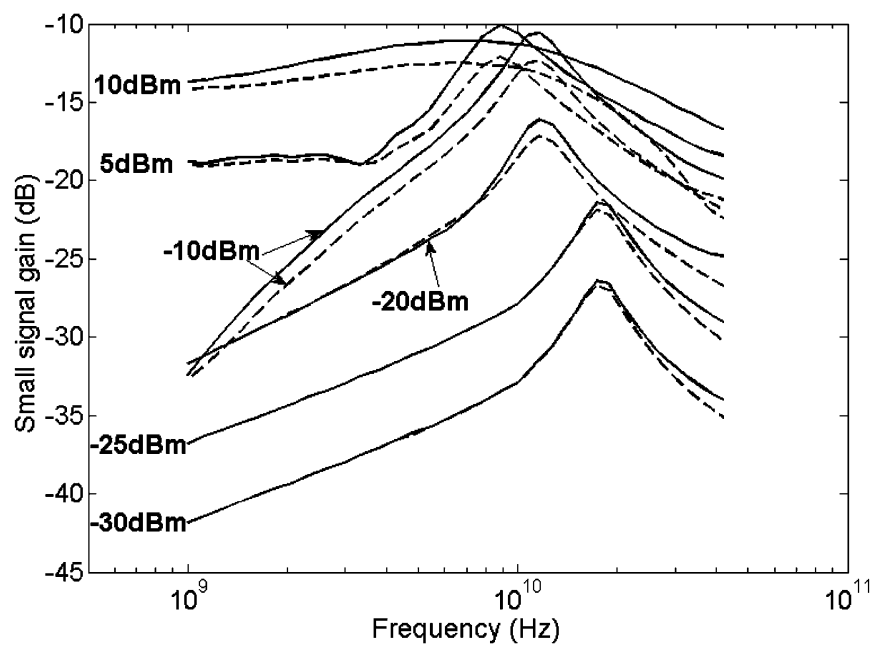

(b)

Fig. 6. Simulated crosstalk induced on a weak probe signal of $-50 \mathrm{dBm}$ in the counter-propagation regime for different $\mathrm{CW}$ input powers in the case of (a) the GCSOA and (b) of the LOA. Full lines and dashed lines correspond to backward and forward propagating probes, respectively.

could be observed between the modulation of the forward and backward propagating laser powers. At higher frequencies we observe a stronger decrease of the backward crosstalk due to the fact that if the components transit time becomes comparable with the period of the signal, the backward propagating probe signal only feels an average of the carrier density change. This walk-off effect has been mentioned before as being a bandwidth limiting factor for wavelength conversion using cross-gain modulation (XGM) in a SOA in the counter-propagating regime [22]. For the case of no remaining laser power we see this even leads to a minimum when half of the period of the input signal is equal to the propagation time in the component $(\approx 12 \mathrm{ps})$.

A discontinuity can also be observed in the case of remaining laser power, at a frequency equal to the inverse of the round trip time $(\approx 2 \times 1400 \mu \mathrm{m})$. This is the result of a resonance in the laser power modulation at this frequency due to the excitation of one of the optical sidemodes of the cavity [23].

Similar simulations for the case of the LOA resulted in crosstalk characteristics as depicted in Fig. 5(b). Again we observe a clear resonance if not being saturated by the input signal. For the forward probe this was also predicted by the small-signal analysis presented in [12] and experimentally observed in [13]. Once saturation sets in, there is a gradual transition from this regime to the low-pass regime of a normal SOA. Detailed inspection of the SOA regime revealed again an overshoot in the characteristic, being slightly higher for the backward probe. In all the cases there only seems to be a difference between both propagation directions at higher frequencies, again caused by the walk-off effect mentioned above. A minimum could again be observed for the SOA case at a signal period equal to half of the propagation time through the saturated part of the component. This resulted in a decrease of the frequency at which the minimum occurs with increasing input power. Although it would not have been possible to be simulated with the given model, a second resonance as was the case for the GCSOA will not be observed in this case due to the much smaller cavity length of the incorporated laser (typically a few microns) rendering a much higher second-order resonance frequency. The differences between forward and backward crosstalk observed at lower frequencies in the case of the GCSOA are not present in this case. This stems from the local coupling between the (local) laser field and the signal in the LOA, whereas in the GCSOA there is a global coupling between the $z$-dependent laser field and the $z$-dependent signal.

The same tests were repeated for the counter-propagating regime discussed in Section III. The results for the GCSOA case are given in Fig. 6(a). As long as a significant amount of laser power is present in the cavity there exists almost no difference in crosstalk as compared to the copropagating regime studied above. At input powers in the vicinity of the input saturation power, both regimes begin to show significant differences, with overall a smaller crosstalk for the counter-propagating case, mainly caused by the lower degree of saturation in the part of the amplifier where the amplitude of the signal becomes the biggest. At higher frequencies however, the walk-off effect discussed above tends to be less pronounced. This could also be seen in the SOA case, where the previously mentioned minima are not that strong any more since in parallel with the weak forward propagating probe signal, there is also the strong $\mathrm{CW}$ signal. It should be noted that now the overshoot in the SOA regime is more explicit as compared to the previous case. This could be understood by noting that in [21] it was stated that for the counter-propagating case an overshoot exists regardless of the magnitude of the waveguide loss. We can also see that due to this overshoot the XGM 3-dB bandwidth is improved in this case for both propagation directions, as compared to copropagating case, be it with a smaller efficiency.

Fig. 6(b) shows the results for the crosstalk as induced in a LOA in the counter-propagating injection scheme. In the case of input powers where almost no saturation exists, characteristics with almost the same peak value of the copropagating scheme are obtained, but with a higher resonance frequency since the resonance now originates mainly in the front part, where still a lot of laser power is still present. If the input power is further increased we find the combination of the low-pass characteristic of a SOA with increasing length (thus with an increasing 


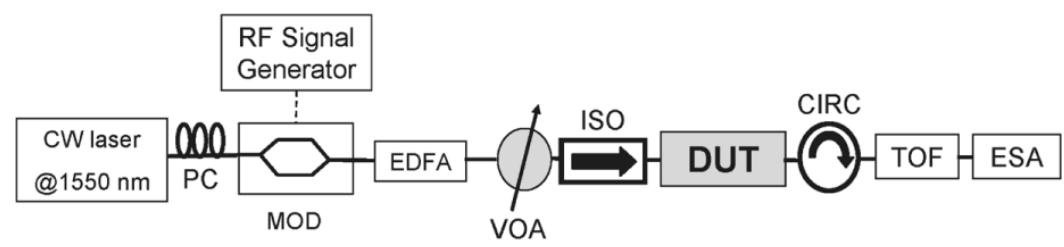

(a)

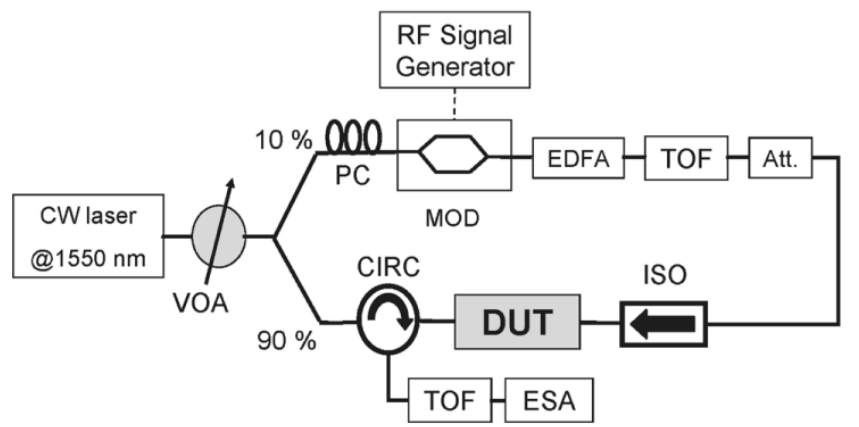

(b)

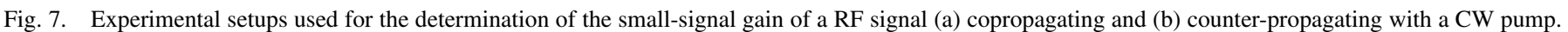

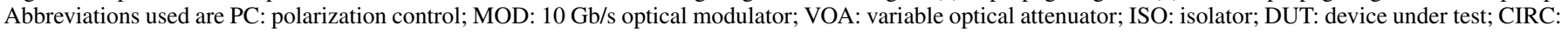
circulator; TOF: tunable optical filter; ESA: electrical spectrum analyzer; Att.: Attenuator.

3-dB bandwidth) and the resonance behavior of the laser part with a decreasing resonance frequency. This could be seen by inspecting the curves for a lot of input powers. This resonance contribution stays relatively important over a larger input power range as was the case in the copropagation scheme since now the remaining laser power is present in the part were the amplitude of the variation is bigger. Once we have no more laser power left, we obtain the low-pass characteristic of a normal SOA with a significant overshoot.

\section{EXPERIMENTAL SETUP AND RESULTS}

\section{A. Experimental Setup}

Small-signal modulation experiments for the co- and counter-propagation regimes were performed using the setups as schematically depicted in Fig. 7(a) and (b), respectively. In both cases the input signal was generated by a tunable laser emitting at $1550 \mathrm{~nm}$.

To perform the co-propagation experiments the input signal was modulated with a very small amplitude $(\approx 10 \%$ of the dc component of the signal). The modulation frequency of this input signal could be swept with a signal generator driving the $10 \mathrm{~Gb} / \mathrm{s}$ modulator. Consequently, reliable measurements up to a modulation frequency of approximately $10 \mathrm{GHz}$ could be performed. After an extra amplification with an erbium-doped fiber amplifier (EDFA), the signal was sent through a variable optical attenuator used to sweep the mean input signal power to the device under test (DUT). In this way a constant ratio between amplitude and $\mathrm{CW}$ value was ensured during the different measurements. The output signal was filtered and detected by an electrical spectrum analyzer. To obtain the final small-signal gain characteristic, the output amplitudes of the data signal were scaled on the input amplitude at each frequency, thereby eliminating the modulation characteristic of the modulator used.

In the counter-propagation regime, things were little different. The input signal was split now before the modulator, and only a small part was modulated. The amplitude of the modulation was in this case little less then the dc component. It was inspected precisely that no higher harmonics were present in the input signal, to ensure the elimination of higher order influences on the small-signal gain of the given signal frequency. An extra filter was used after the modulator to eliminate the noise addition at low input powers. An extra attenuator was inserted to tune the dc component of the modulated input signal to a tenth of the CW input signal injected in the front. A circulator in combination with a tunable optical filter (TOF) was now used to select the backward propagating signal.

For the crosstalk measurements an extra probe signal with an amplitude of $-25 \mathrm{dBm}$ was injected, generated by a DFB laser diode emitting at $1542 \mathrm{~nm}$. To measure the forward and backward crosstalk in the co- and counter-propagation setup, respectively, this could be simply done by adding the probe to the signal path, and to retune the TOF to the probe wavelength. In order to measure the crosstalk in the opposite direction of the modulated signal, the circulator and the isolator must be switched place. The crosstalk was in any case measured by the electrical spectrum analyzer (ESA) as the ratio of the amplitude of the induced modulation to the average detected power.

The GCSOA used during the experiments was a commercially available component acquired from Alcatel [1] some years ago. The lasing wavelength was $1508 \mathrm{~nm}$. During the experiments the GCSOA was driven with $120 \mathrm{~mA}$. It had a de gain of $18 \mathrm{~dB}$ for the given signal wavelength. It should be noted that the probe and signal wavelength were both sufficiently detuned from the lasing wavelength of the GCSOA used during the experiments to avoid injection locking [24]. The LOA used was a prototype fabricated by Genoa, [2]. It was driven at $150 \mathrm{~mA}$. It had a dc gain of $16.5 \mathrm{~dB}$ for the signal wavelength.

\section{B. Small-Signal Gain Measurements}

Fig. 8(a) and (b) depicts the small-signal amplification characteristics resulting from the co-propagation setup for some dif- 


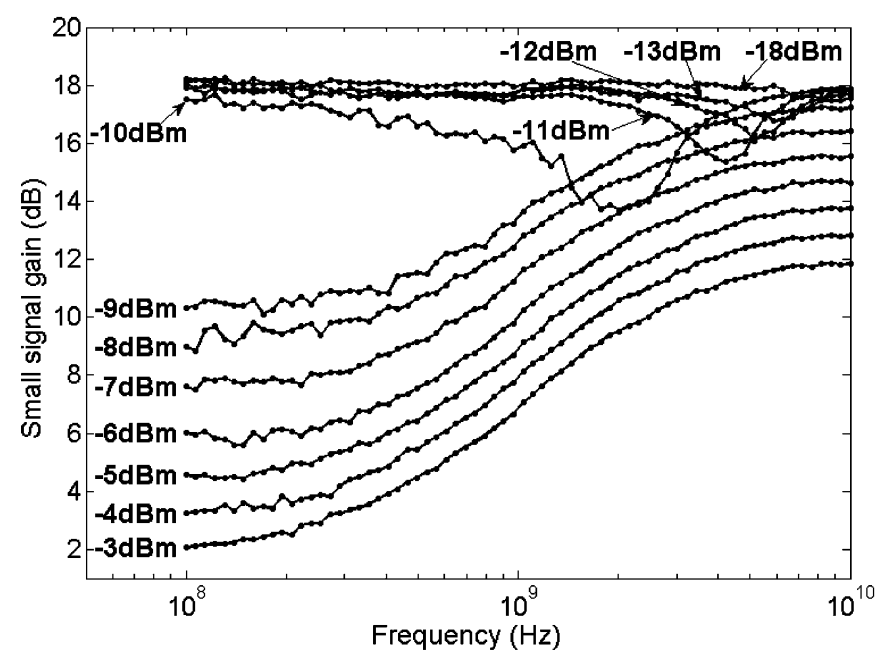

(a)

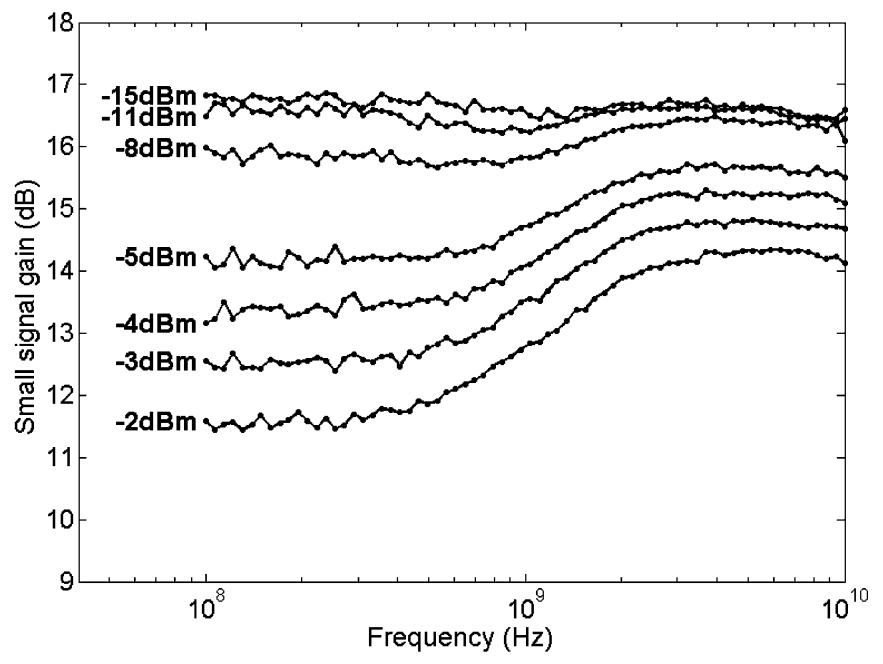

(b)

Fig. 8. Experimentally obtained small-signal gain curves of a (a) GCSOA driven with $100 \mathrm{~mA}$ and of (b) a LOA driven with $150 \mathrm{~mA}$ in the co-propagation regime.

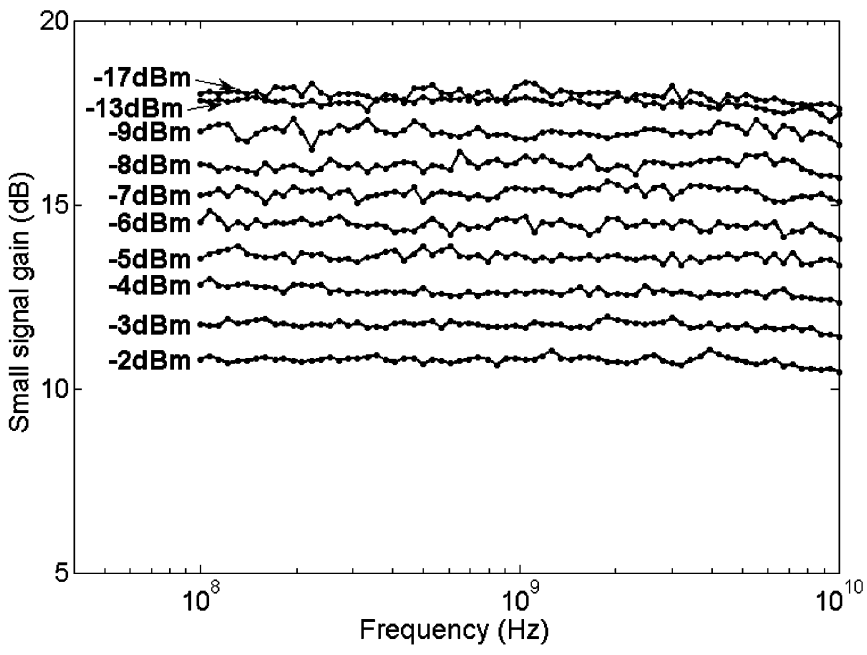

(a)

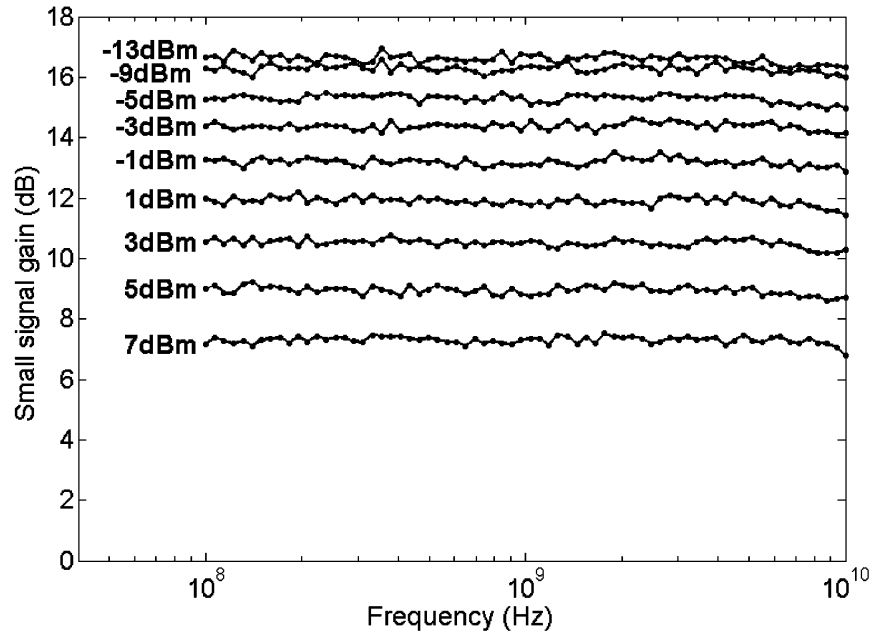

(b)

Fig. 9. Experimentally obtained small-signal gain curves of a (a) GCSOA driven with $100 \mathrm{~mA}$ and of (b) a LOA driven with $150 \mathrm{~mA}$ in the counter-propagation regime.

ferent input powers, in the case of a GCSOA and a LOA, respectively. For clarity reasons in the case of the LOA only a relatively small input power region is shown. This makes it possible to see the very weak resonance effect at an input power of $-11 \mathrm{dBm}$. It can be seen that for low input powers the gain is almost equal to the dc gain for all signal frequencies. The experimentally obtained SSFR of the GCSOA show a much more explicit resonance phenomenon as was predicted by the simulations. The input saturation power tends to be approximately $-9.5 \mathrm{dBm}$. A sudden switch to the high-pass SOA characteristic can be observed once this power is exceeded, in contrast with a more gradual transition in the LOA. It should be noted that the resonance frequency of the LOA is little more then $1 \mathrm{GHz}$, whereas this was much higher (somewhat smaller then $10 \mathrm{GHz}$ ) in the simulations. In measurements of [13] a resonance of almost $3 \mathrm{GHz}$ was observed, whereas the model of [12] also resulted in some cases in resonance frequencies in the vicinity of $10 \mathrm{GHz}$. However, this absolute value is not that important in understanding the effects discussed here. Of course the lower resonance frequency leads to much smaller crosstalk at higher bitrates, which is advantageous in WDM applications.

The results for the counter-propagation regime are shown in Fig. 9(a) and (b) for the GCSOA and the LOA, respectively. A fairly constant amplification is obtained, within the given measurement accuracies. This is in good agreement with the numerical results. By comparison with the co-propagation measurement we see that the obtained constant gain is almost equal to the high frequency limit of the previous case, with a small deviation in the saturated case possibly caused by a little consumption of the dc gain by the counter-propagating modulated signal.

\section{Small-Signal Crosstalk Measurements}

The experimentally obtained crosstalk induced on the probe signal in forward and backward propagation direction in the co-propagation scheme are plotted both in the same figure again to make comparison possible. For the GCSOA this is shown in 


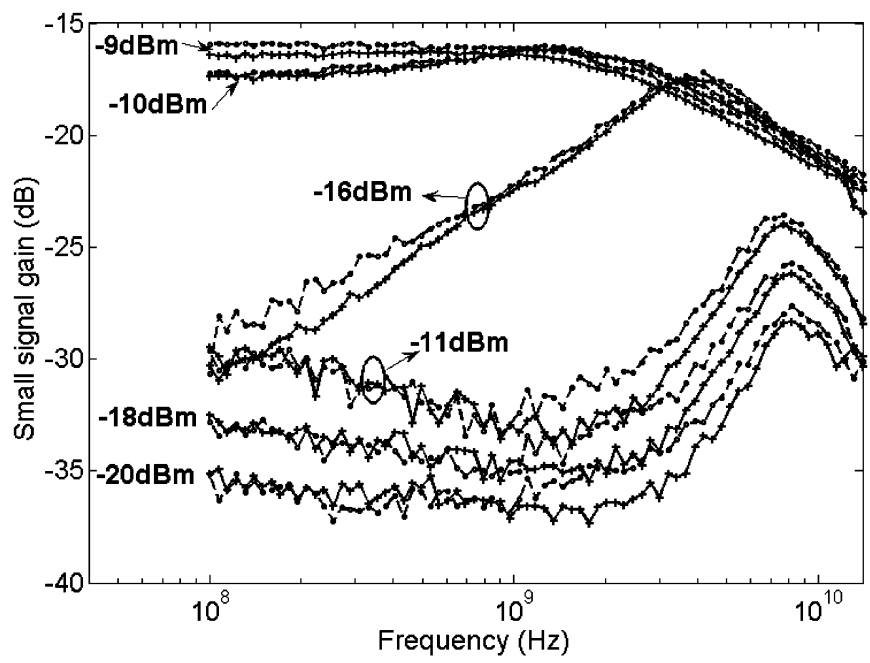

(a)

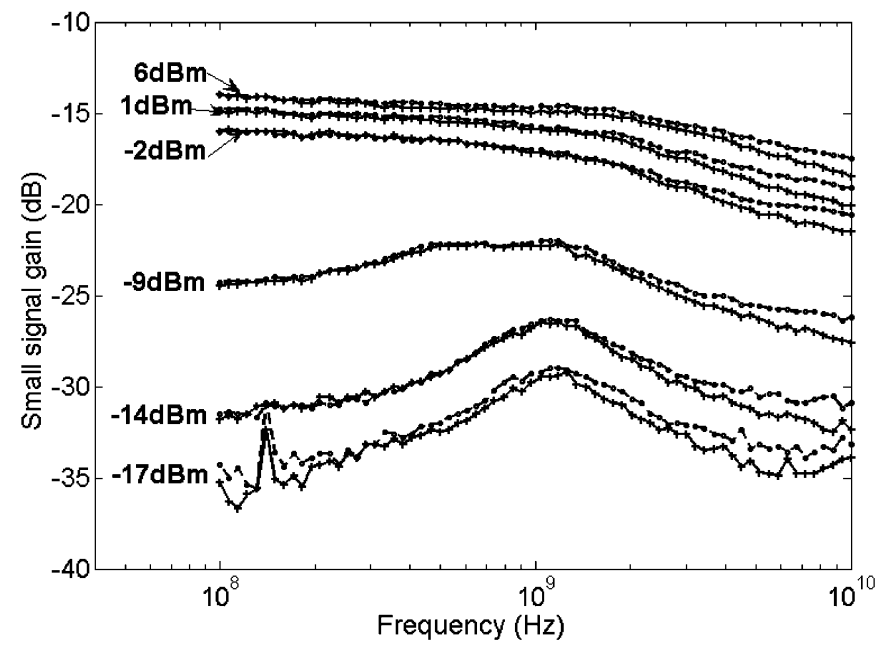

(b)

Fig. 10. Experimentally obtained crosstalk in the co-propagation setup of a forward and a backward probe of $-25 \mathrm{dBm}$ with (a) the GCSOA driven with 100 $\mathrm{mA}$ and (b) the LOA driven with $150 \mathrm{~mA}$. Dashed line with crosses: backward probe. Full line with circles: forward probe.

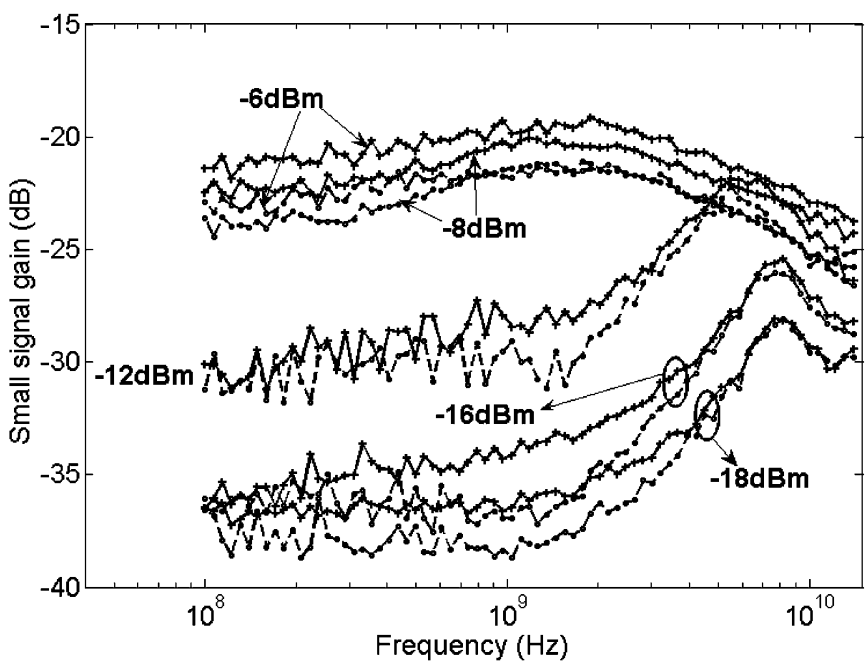

(a)

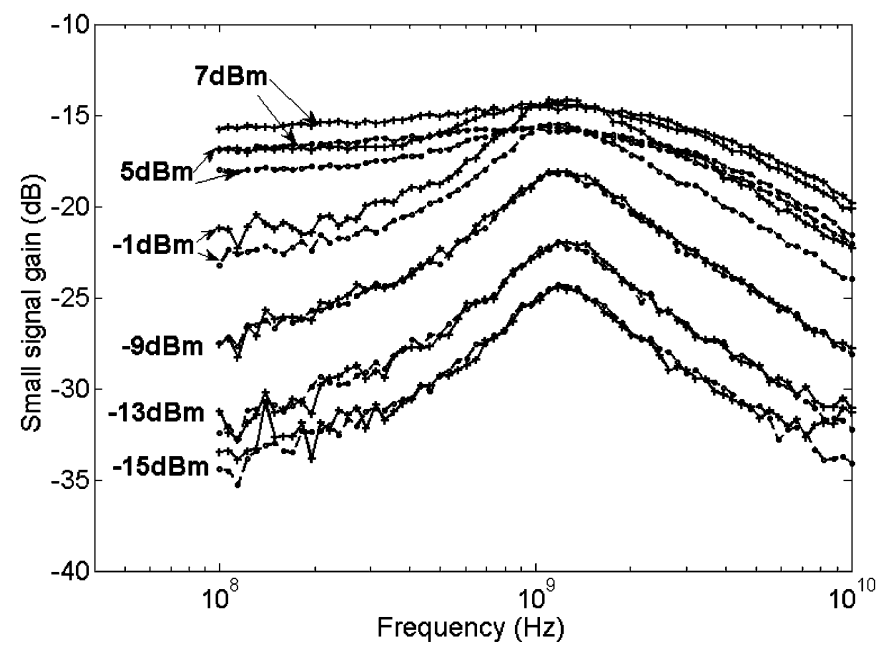

(b)

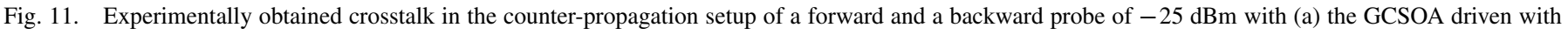
$100 \mathrm{~mA}$ and (b) the LOA driven with $150 \mathrm{~mA}$. Dashed line with crosses: backward probe. Full line with circles: forward probe.

Fig. 10(a), whereas the LOA results are depicted in Fig. 10(b). Once more there are only depicted a few curves in order to keep the figure surveyable.

For the LOA, we see a very good agreement with the simulations. We observe again a gradual evolution from the purely laser related resonance behavior, to the pure SOA low-pass characteristic. In between we observe again the combination of both. The difference in slope of the forward and backward induced crosstalk indicates the presence of the walk-off effect mentioned in Section IV. The minimum could of course not be observed since it is located at much higher frequencies.

In the case of the GCSOA, somewhat less accurate measurements were obtained in the low frequency range, however they still were suitable to illustrate the most important trends. Detailed inspection showed again the difference in slope between forward and backward propagating crosstalk in the saturated case. At low input powers we can clearly see the explicit resonance. The deviation between backward and forward propagation in the low frequency is somewhat less explicit as was the case in the numerical study however still visible.

The forward and backward crosstalk measured in both components in the counter-propagation regime is depicted in Fig. 11(a) (GCSOA) and Fig. 11(b) (LOA).

The LOA measurements do agree rather good with the numerical results. The increase of the slope at low frequencies for input powers in the transition zone is less pronounced here due to the lower resonance frequency, thereby not differing much from the cut-off frequency of the SOA low-pass characteristic. Further we can again observe the more explicit overshoot in the SOA characteristic as compared to the forward case. The walk-off effect on the other hand is less pronounced than was predicted by the numerical study. 
Results obtained with the GCSOA tend to be a little less accurate, but as was the case above show most of the trends discussed above.

\section{CONCLUSION}

We performed a systematic and in-depth numerical and experimental analysis of the dynamics of two types of gain-clamped semiconductor amplifiers under small-signal optical modulation. A rate equation model enabling numerical studies of the dynamics in a LOA was developed.

A study of the small-signal gain of a RF signal co- and counter-propagating with a strong pump resulted in the observation of a clear resonance phenomenon in the co-propagating case leading to a dip in the SSFR. The dip seems to occur at a decreasing frequency in function of the injected $\mathrm{CW}$ power. This was less pronounced in the LOA due to the clamping being the result of several independently acting lasers each providing only a small part of the gain. In the counter-propagating case we found a fairly constant amplification in function of signal frequency for both amplifiers. This was the result of the less pronounced modulation of the input signal in the part of the amplifiers being most sensitive to power variations. The numerically obtained results were confirmed by experiments.

In both the co- and counter-propagation regime, the crosstalk induced on a forward and backward injected probe signal were studied. Simulations as well as experiments showed a clear resonant behavior in both components as long as laser light was present in the cavity. With increasing input power, this resulted in the LOA in a gradual transition from this regime to a low-pass behavior of a SOA. In the GCSOA, this transition took place in a smaller input power region. A walk-off effect resulted in both components in a faster decrease of the backward as compared to the forward crosstalk for high frequencies in the co-propagation regime. In the counter-propagation case, this was somewhat less pronounced. Also in the lower frequency range, a difference between the crosstalk on a backward propagating and a forward propagating probe could be observed in the GCSOA due to a phase delay associated with signal, laser and probe, all propagating in parallel.

It is clear that the difference in clamping mechanism results in a clearly different RF signal amplification behavior. The crosstalk, on the other hand, is behaving much more similar in both cases, showing a clear resonance in the vicinity of the resonance frequencies of the incorporated lasers. In the experiments those resonance frequencies seemed to be much smaller for the LOA. The study also showed that for most applications both components can be used best at low saturation degrees, thereby avoiding explicit resonance effects.

\section{REFERENCES}

[1] P. Doussiere, A. Jourdan, G. Soulage, P. Garabedian, C. Graver, T. Fillion, E. Derouin, and D. Leclerc, "Clamped gain travelling wave semiconductor optical amplifier for wavelength division multiplexing applications," in Proc. Int. Semiconduct. Laser Conf., Sep. 1994, pp. 185-186.

[2] D. Francis, S. DiJaili, and J. Walker, "A single chip linear optical amplifier," in Proc. Opt. Fiber Commun. Conf., Mar. 2001, p. PD13.
[3] L. Tiemeijer, P. Thijs, T. van Dongen, J. Binsma, E. Jansen, and H. van Helleputte, "Reduced intermodulation distortion in $1300 \mathrm{~nm}$ gainclamped MQW laser amplifiers," IEEE Photon. Technol. Lett., vol. 7, no. 3, pp. 284-286, Mar. 1995.

[4] J. C. E. Tangdiongga, L. Spiekman, G. van den Hoven, and H. de Waardt, "Performance analysis of linear optical amplifiers in dynamic WDM systems," IEEE Photon. Technol. Lett., vol. 14, no. 8, pp. 1196-1198, Aug. 2002.

[5] H. Lee, J. Oh, D. Lee, G. Lee, and S. Hwang, "Demonstration of $16 \times$ $10 \mathrm{~Gb} / \mathrm{s}$ WDM transmission over $4 \times 40 \mathrm{~km}$ of SMF using linear optical amplifiers combined with Raman-pumped dispersion compensation fibers under dynamic add/drop situations," IEEE Photon. Technol. Lett., vol. 16, no. 6, pp. 1576-1578, Jun. 2004.

[6] G. Morthier, M. Zhao, B. Vanderhaegen, and R. Baets, "Experimental demonstration of an all-optical $2 R$ regenerator with adjustable decision threshold and "true" regeneration characteristics," IEEE Photon. Technol. Lett., vol. 12, no. 11, pp. 1516-1518, Nov. 2000.

[7] M. Zhao, J. D. Merlier, G. Morthier, and R. Baets, "All-optical $2 R$ regeneration based on polarization rotation in a linear optical amplifier," IEEE Photon. Technol. Lett., vol. 15, no. 2, pp. 305-307, Feb. 2003.

[8] E. Tangdiongga, J. Turkiewicz, G. Khoe, and H. de Waardt, "Clock recovery by a fiber ring laser employing a linear optical amplifier," IEEE Photon. Technol. Lett., vol. 16, no. 2, pp. 611-613, Feb. 2004.

[9] J. Sun, G. Morthier, and R. Baets, "Numerical and theoretical study of the crosstalk in gain-clamped semiconductor optical amplifiers," IEEE J. Sel. Topics Quantum Electron., vol. 3, no. 5, pp. 1162-1167, Sep./Oct. 1997.

[10] X. Jin, T. Keating, and S. L. Chuang, "Theory and experiment of highspeed cross-gain modulation in semiconductor lasers," IEEE J. Quantum Electron., vol. 36, no. 12, pp. 1485-1493, Dec. 2000.

[11] J. Oksanen and J. Tulkki, "On crosstalk and noise in an optical amplifier with gain clamping by vertical laser field," J. Lightw. Technol., vol. 21, no. 9, pp. 1914-1919, Sep. 2003.

[12] C.-Y. Jin, Y.-Z. Huang, L.-J. Yu, and S.-L. Deng, "Numerical and theoretical analysis of the crosstalk in linear optical amplifiers," IEEE $J$. Quantum Electron., vol. 41, no. 5, pp. 636-641, May 2005.

[13] D. Tong, M. Wu, and S. Dijaili, "Intrinsic intermodulation distortion characteristics of linear optical amplifier," in Proc. Conf. Lasers and Electro-Optics, Jun. 2003, pp. 1414-1416.

[14] C.-Y. Jin, Y.-Z. Huang, L.-J. Yu, and S. Deng, "Detailed model and investigation of gain saturation and carrier spatial hole burning for a semiconductor optical amplifier with gain clamping by a vertical laser field," IEEE J. Quantum Electron., vol. 40, no. 5, pp. 513-518, May 2004.

[15] L. Schares, C. Schubert, C. Schmidt, H. G. Weber, L. Occhi, and G. Guekos, "Phase dynamics of semiconductor optical amplifiers at 10-40 GHz," IEEE J. Quantum Electron., vol. 39, no. 11, pp. 1394-1408, Nov. 2003.

[16] M. Asghari, I. H. White, and R. V. Penty, "Wavelength conversion using semiconductor optical amplifiers," J. Lightw. Technol., no. 7, pp. 1181-1190, Jul. 1997.

[17] K. Petermann, Laser Diode Modulation and Noise. Norwell, MA: Kluwer, 1988.

[18] P. Vankwikelberge, G. Morthier, and R. Baets, "Cladiss-a longitudinal multimode model for the analysis of the static, dynamic, and stochastic behavior of diode lasers with distributed feedback," IEEE J. Quantum Electron., vol. 26, no. 10, pp. 1728-1741, Oct. 1990.

[19] G. Morthier, J. Sun, T. Gyselings, and R. Baets, "A novel optical decision circuit based on a Mach-Zehnder or Michelson interferometer and gain-clamped semiconductor optical amplifiers," IEEE Photon. Technol. Lett., vol. 10, no. 8, pp. 1162-1164, Aug. 1998.

[20] T. Durhuus, B. Mikkelsen, C. Joergensen, S. Danielsen, and K. Stubkjaer, "All-optical wavelength conversion by semiconductor optical amplifiers," J. Lightw. Technol, vol. 14, no. 6, pp. 942-954, Jun. 1996.

[21] M. Nielsen, D. Blumenthal, and J. Mørk, "A transfert function approach to the small-signal response of saturated semiconductor optical amplifiers," J. Lightw. Technol, vol. 18, no. 12, pp. 2151-2157, Dec. 2000.

[22] D. Marcenac and A. Mecozzi, "Switches and frequency converters based on cross-gain modulation in semiconductor optical amplifiers," IEEE Photon. Technol Lett., vol. 9, no. 6, pp. 749-751, Jun. 1997.

[23] G. Morthier, R. Schatz, and O. Kjebon, "Extended modulation bandwidth of DBR and external cavity lasers by utilizing a cavity resonance for equalization," IEEE J. Quantum Electron., vol. 36, no. 12, pp. 1468-1475, Dec. 2000.

[24] V. Annovazzi-Lodi, A. Scire, M. Sorel, and S. Donati, "Dynamic behavior and locking of a semiconductor laser subjected to external injection," IEEE J. Quantum Electron., vol. 34, no. 12, pp. 2350-2357, Dec. 1998. 


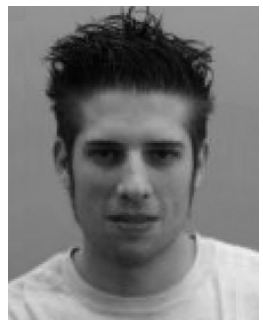

Sam Verspurten (S'01) was born in Ghent, Belgium, in 1978. He received the M.S. degree in electrotechnical engineering from Ghent University, Ghent, Belgium, in 2001, where he is currently working toward the $\mathrm{Ph} . \mathrm{D}$. degree in electrical engineering.

His research interests include all-optical signal processing using semiconductor optical amplifiers and the modeling of the dynamics of those components.

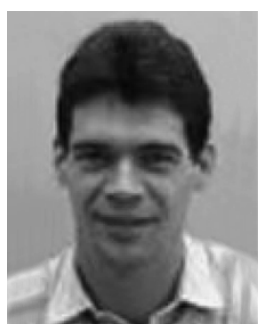

Geert Morthier (M'93-SM'01) received the M.S. degree in electrical engineering and the Ph.D. degree from Ghent University, Ghent, Belgium, in 1987 and 1991, respectively.

Since 1991, he has been a Member of the Permanent Staff of the Interuniversity MicroElectronics Center (IMEC), Ghent University. From 1998 to 1999, he was the Project Manager of the ACTS project ACTUAL, and since 2001 he has been Project Manager of the IST project NEWTON on widely tunable lasers. In 2001, he was appointed part-time Professor at Ghent University. He has authored or coauthored around 100 papers and two books in the field. His main research interests are in the modeling and characterization of optoelectronic components.

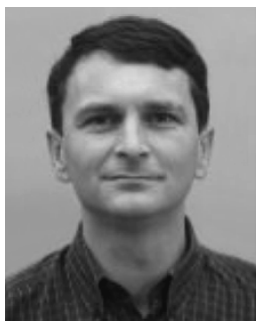

Roel Baets (M'88-SM'96) received the M.S. degree in electrical engineering and the Ph.D. degree from Ghent University, Ghent, Belgium, in 1980 and 1984, respectively, and the M.Sc. degree in electrical engineering from Stanford University, Stanford, CA, in 1981.

Since 1981, he has been with the Department of Information Technology (INTEC), Ghent University, where he has been a Professor since 1989. He is currently the Head of the optoelectronics group. $\mathrm{He}$ has worked in the field of III-V devices for optoelectronic systems. With about 200 publications and conference papers, he has made contributions to the design and fabrication of semiconductor laser diodes, passive guided wave devices, PICs, and microoptic components.

Dr. Baets is a Member of the Optical Society of America, the IEEE Laser and Electro-Optics Society, the International Society for Optical Engineering (SPIE), and the Flemish Engineers Association. 